\title{
INFRARED EMISSION IN SEYFERT 2 GALAXIES: REPROCESSED RADIATION FROM A DUSTY TORUS?
}

\author{
Thaisa Storchi-BergmanN \\ Instituto de Fisica, UFRGS, Campus do Vale, 91500 Porto Alegre, RS, Brazil; and \\ Astronomy Department, University of Maryland \\ AND \\ JoHn S. MulchaeY AND ANDrew S. Wilson \\ Astronomy Department, University of Maryland; and Space Telescope Science Institute, 3700 San Martin Drive, Baltimore, MD 21218 \\ Received 1992 April 28; accepted 1992 June 4
}

\begin{abstract}
The nature of the infrared continuum in Seyfert 2 galaxies is not well understood. An attractive model is one in which dust grains near the nucleus are heated by optical and ultraviolet photons from a central source and reradiate in the IR. However, a comparison of the observed optical-UV continuum with the observed IR emission indicates that in many Seyfert 2's there is a deficiency of dust heating photons. This apparent deficiency may be understood if the photons responsible for heating the dust escape anisotropically. Recent work has revealed that a number of Seyfert 2 galaxies exhibit conically shaped regions of gas apparently illuminated by a collimated, nuclear ionizing source. In this Letter, we test one model for this collimation, namely that the cones result from shadowing of a compact nuclear continuum source by a thick, dusty torus. From the emission-line ratios measured for gas within the cones, we have calculated the number of ionizing photons emitted by the compact nucleus. Then, on the assumption that this compact nuclear source radiates isotropically, we have found the optical-UV power incident on the torus, which is expected to be reradiated in the IR. We find the observed IRAS luminosities are consistent with the torus model in eight of the nine objects with sufficient data to perform the calculation.
\end{abstract}

Subject headings: galaxies: active — galaxies: nuclei — galaxies: Seyfert — infrared: galaxies

\section{INTRODUCTION}

An outstanding problem in our understanding of Seyfert galaxies has been the source of the infrared continuum in these objects. Thermal emission from dust heated by a compact optical-ultraviolet source is the most attractive model. However, the observed optical-UV continuum is much weaker than the observed thermal IR emission in many Seyfert 2 galaxies. This implies that either extranuclear sources contribute dust heating photons or the optical-UV radiation escapes anisotropically (e.g., Wilson, Ward, \& Haniff 1988).

There is now considerable evidence that ionizing photons escape from the nuclear source anisotropically in many Seyfert 2 galaxies (see, e.g., Wilson 1992 for a recent review). For some objects, this anisotropy becomes evident in the elongated morphologies detected in images taken through narrow-band filters centered in high-excitation lines (e.g., [O III] 25007) or through "ionization maps" (e.g., Pogge 1988a, b)-ratios between the continuum-subtracted images in [O III] $\lambda 5007$ and in $\mathrm{H} \alpha+[\mathrm{N}$ II $] \lambda \lambda 6548,6583$. In about 10 nearby Seyfert 2's, these ratio maps show a clear conical or biconical morphology.

The presence of these "ionization cones" is consistent with the "Unified Scheme" for Seyfert galaxies (Antonucci \& Miller 1985; Krolik \& Begelman 1986), in which the conical morphology results from shadowing by a dusty obscuring torus, which hides the nuclear source and broad-line region from direct view. Another possible source of collimation is the intrinsic anisotropy of a thick accretion disk (e.g., Madau 1988; AcostaPulido et al. 1990). The obscuring torus model, however, may more naturally account for the IR observations.

In this Letter, we combine new and existing data for a sample of nine Seyfert 2 galaxies with known “ionization cones," in order to test whether the collimation results from shadowing of radiation from a small, isotropic, nuclear source by a thick dusty torus. From long-slit spectroscopic data, the emission-line properties of the gas in the cones are obtained and used to calculate the intrinsic luminosity of the ionizing source. Narrow-band images are used to obtain the opening angles of the cones which together with the intrinsic opticalUV luminosity of the source, allow the IR luminosity of the torus to be predicted. This predicted luminosity is then compared with the observed IR luminosities of the galaxies calculated from the IRAS fluxes.

\section{THE SAMPLE}

Our sample comprises galaxies with Seyfert 2 nuclei and ionization cones, for which long-slit spectra with good spatial resolution are available. The galaxies are listed in Table 1, where we also list the morphological types taken from the Third Reference Catalogue of Galaxies (col. [2]), adopted distances (for $H_{0}=75 \mathrm{~km} \mathrm{~s}^{-1} \mathrm{Mpc}^{-1}$; col. [3]), and references for the detection of the conical morphology (col. [4]) and longslit spectra (col. [5]) used to derive the parameters necessary to predict the torus luminosity. Although the galaxies NGC 1068, NGC 1365, NGC 4388, and NGC 5728 have star-forming regions near the nucleus, we used only spectra avoiding these regions. For NGC 1068 we used the highest excitation spectra from the [O III] "plume" and from a region in the same direction farther away, while for NGC 1365 we used a spectrum from the SE cone region, which appears to be uncontaminated by the strongest star-forming regions. In NGC 4388, we use the spectra with reliable fluxes as far from the galaxy disk as possible, while in NGC 5728 we used only the high-excitation 
TABLE 1

BASIC DATA

\begin{tabular}{|c|c|c|c|c|}
\hline $\begin{array}{l}\text { Galaxy } \\
\text { (1) }\end{array}$ & $\begin{array}{l}\text { Type } \\
(2)\end{array}$ & $\begin{array}{l}D(\mathrm{Mpc}) \\
\quad(3)\end{array}$ & $\begin{array}{l}\text { Conical Morphology } \\
\text { (4) }\end{array}$ & $\begin{array}{l}\text { Long-Slit Spectroscopy } \\
(5)\end{array}$ \\
\hline NGC $1068 \ldots \ldots$. & $\mathrm{SA}(\mathrm{rs}) \mathrm{b}$ & 15 & Pogge 1988a & $\begin{array}{l}\text { Bergeron, Petitjean, \& Durrett 1989; } \\
\text { Baldwin, Wilson, \& Whittle } 1987\end{array}$ \\
\hline NGC $1365 \quad \ldots . .$. & $\mathrm{SBb}$ & 20 & $\begin{array}{l}\text { Phillips et al. 1983; Storchi-Bergmann \& } \\
\text { Bonatto } 1991\end{array}$ & Edmunds \& Pagel 1982 \\
\hline NGC $2110 \ldots \ldots$. & SAB0 & 30 & Pogge 1989; Haniff, Wilson, \& Ward 1991 & Wilson, Baldwin, \& Ulvestad 1985 \\
\hline NGC $3281 \ldots \ldots$. & $\mathrm{SAab}(\mathrm{pec})$ & 42 & Storchi-Bergmann, Wilson, \& Baldwin 1992 & Storchi-Bergmann, Wilson, \& Baldwin 1992 \\
\hline NGC $4388 \ldots . .$. & $\mathrm{SAb}$ & 18 & $\begin{array}{l}\text { Pogge 1988b, 1989; Corbin, Baldwin, \& } \\
\text { Wilson } 1988\end{array}$ & Colina 1992 \\
\hline NGC $5506 \ldots \ldots$. & $\mathrm{Sa}(\mathrm{pec})$ & 23 & Wilson, Baldwin, \& Ulvestad 1985 & $\begin{array}{l}\text { Wilson, Baldwin, \& Ulvestad 1985; } \\
\text { Veilleux } 1991\end{array}$ \\
\hline NGC $5728 \ldots \ldots$. & SABa & 36 & Pogge 1989 & Schommer et al. 1988 \\
\hline Mrk $78 \ldots \ldots \ldots$ & E & 149 & Pedlar et al. 1989 & Mulchaey, Wilson, \& Whittle 1992 b \\
\hline Mrk $573 \ldots \ldots \ldots$ & $\mathrm{SAB}(\mathrm{rs}) 0$ & 69 & Haniff, Wilson, \& Ward 1991 & Tsvetanov \& Walsh 1992 \\
\hline
\end{tabular}

spectra from the "fan-shaped" structure to the SE of the nucleus.

\section{THE CALCULATIONS}

Our goal is to predict the luminosity of the torus assuming the ionizing source is an isotropic radiator, the ionization cones represent the free solid angle, and the torus absorbs all photons in the wavelength range $100 \AA-1 \mu \mathrm{m}$. The torus is expected to reradiate this energy in the infrared (Krolik \& Lepp 1989). We first calculate the number of ionizing photons emitted from the central source using the emission-line ratios in the cone. Then, by assuming a spectral shape, the total central source luminosity in the $100 \AA-1 \mu \mathrm{m}$ band can be calculated, as well as the fraction incident on the torus. The luminosity incident on the torus is assumed to be reradiated isotropically and can then be compared with the IR luminosity determined from the IRAS fluxes. If such a dusty torus is indeed present, the observed IR luminosity should be equal to or larger than the calculated luminosity (because of the large $I R A S$ beam, extranuclear sources, such as star-forming regions, may contribute to the $I R A S$ flux-see $\S 4$ ). If the calculated luminosity incident on the torus is significantly larger than the observed IR luminosity, then the simple model is inconsistent with the data. In this case, the ionizing source could be intrinsically anisotropic (e.g., Miller, Goodrich, \& Mathews 1991) or the torus itself could be optically thick even at mid-infrared wavelengths (Pier and Krolik 1992). The steps followed in the calculations are as follows:

1. The number of ionizing photons $Q$ is calculated from the ionization parameter $U$ :

$$
Q=U 4 \pi r^{2} n c,
$$

where $n$ is the gas density in the cone at a distance $r$ from the ionizing source. $U$ is obtained by comparing the measured emission-line ratios in the cone region with photoionization model calculations from the code CLOUDY (Ferland 1991). For all objects, the emission line ratios of $[\mathrm{O} \mathrm{III}] \lambda 5007 / \mathrm{H} \beta$ and [N II] $\lambda 6583 / \mathrm{H} \alpha$ have both been used to estimate $U$. We emphasize that the emission regions used are well separated from the nuclei, so that the projected separations on the sky are accurately known. To take account statistically of the unknown projection factors, we have multiplied all projected gas-nucleus separations by 1.4 to obtain $r$. The electron density is calculated in the usual way from the $[\mathrm{S}$ II $] \lambda \lambda 6716,6731$ ratio at the point considered. Because the $[\mathrm{S}$ II $]$ lines originate in the partially neutral zone, the electron density so calculated will be less than the true particle density. The electron densities were multiplied by a factor of 1.25 to take account of this effect. This value was obtained from running CLOUDY for nebulae with several gas densities in the range $10^{3}-10^{4} \mathrm{~cm}^{-3}$ and comparing with the electron density obtained from the $[\mathrm{S}$ II $]$ ratio.

2. The luminosity spectrum of the continuum source is taken to be a power law: $L_{v}=A v^{-\alpha}$. Some of the objects have values of $\alpha$ available from IUE observations (Kinney et al. 1991). For the others, we use the typical Seyfert 1 value of 1.5 . The calculation is rather insensitive to the exact choice of $\alpha$. The constant $A$ is then $A=Q h \alpha v_{0}^{\alpha}$, where $v_{0}=3.29 \times 10^{15} \mathrm{~Hz}$.

3. The predicted luminosity of the torus is then

$$
\begin{aligned}
& \qquad L_{P}=(1-C) \int_{v_{1}}^{v_{2}} A v^{-\alpha} d v=(1-C) Q h f(\alpha), \\
& \text { where } f(\alpha)=\alpha v_{0}^{\alpha}\left(v_{2}^{1-\alpha}-v_{1}^{1-\alpha}\right) /(1-\alpha) \text { or } \\
& L_{P}\left(L_{\odot}\right)=6.2 \times 10^{-12}(1-C) U n r_{\mathrm{pc}}^{2} f(\alpha) .
\end{aligned}
$$

Here $v_{2}$ and $v_{1}$ are the assumed upper and lower limits to the frequencies which can heat the dust $\left(v_{2}=3 \times 10^{16} \mathrm{~Hz}=100 \AA\right.$ and $\left.v_{1}=3 \times 10^{14} \mathrm{~Hz}=1 \mu \mathrm{m}\right)$. The covering factor of the bicone is $C=1-\cos (\theta / 2)$, where $\theta$ is the full opening angle of the cones. Because almost all galaxies have $\alpha>1$, the value of $L_{P}$ is insensitive to $v_{2}$, i.e., to the arbitrary choice of $100 \AA$ as the shortest wavelength which heats the dust.

4. This predicted luminosity, $L_{P}$, can be compared to the observed IR luminosity, $L_{\mathrm{IR}}$, calculated as

$$
L_{\mathrm{IR}}\left(L_{\odot}\right)=3.1 \times 10^{16} D_{\mathrm{Mpc}}^{2} F_{\mathrm{IR}},
$$

where $D_{\mathrm{Mpc}}$ is the distance of the galaxy in Mpc (Table 1) and $F_{\text {IR }}$ is the infrared flux in the range $10-120 \mu \mathrm{m}$ in ergs $\mathrm{cm}^{-2}$ $\mathrm{s}^{-1}$, calculated from the IRAS fluxes as in Rowan-Robinson \& Crawford (1989). For all objects we use co-added IRAS fluxes (Mulchaey et al. 1992c).

\section{CONTRIBUTION OF EXTRANUCLEAR SOURCES TO THE IRAS LUMINOSITIES}

Before comparing the predicted torus luminosity with the IR observations, the contribution of the galaxy to the far-infrared flux must be considered. Qualitatitively, it is possible to use IR colors to check the nature of the emission. A plot of the IR spectral index $\alpha(60,25)$ versus $\alpha(100,60)$ for the sample gal- 
axies shows that most of them occupy the region characteristic of Seyferts, while NGC 2110, NGC 1365, and NGC 5728 are in the region overlapped by Seyfert 2's, nuclear H II regions and normal galaxy disks (cf. Miley, Neugebauer, \& Soifer 1985). For NGC 1365 this result is expected because the starburst component is dominant, but in NGC 2110 there is no evidence of a young stellar population in the optical spectra. In the case of NGC 5728, there is a ring of $\mathrm{H}$ II regions surrounding the nucleus, which probably contributes significantly to the infrared emission.

A more quantitative estimate of the contribution of extranuclear sources to the IR luminosities is obtained by using ground-based observations to decompose the observed IR spectrum into nuclear and nonnuclear components. Unfortunately, such a decomposition is only available for one object, NGC 1068. Telesco et al. (1984) find that approximately half of the observed IR emission in this galaxy can be attributed to the Seyfert nucleus. For four other galaxies, Roche et al. (1991) report $12 \mu \mathrm{m}$ fluxes with a beam diameter of $\sim 5^{\prime \prime}(290-680 \mathrm{pc}$ at the assumed distances). From these values, it appears that at least half of the IRAS flux at this wavelength can be attributed to the nuclear source with the exception of NGC 1365 which appears to be dominated by extended emission. Although the ratio of nuclear to nonnuclear flux is probably not the same at longer IRAS wavelengths, the $12 \mu \mathrm{m}$ observations provide a rough correction factor to the $I R A S$ luminosities, and we have multiplied the observed luminosities by this ratio. For the galaxies without sufficient ground-based data, we adopt a correction factor of 0.6 , the median flux ratio Roche et al. (1991) find for Seyfert 2's and LINERS.

\section{RESULTS}

Table 2 shows the relevant quantities and the results of the calculations: distance $r$ from the nucleus at which the line ratios were measured (col. [2]); the negative of the logarithm of the ionization parameter $[-\log (U)]$ obtained from the line ratios (col. [3]); gas density $n$ at the distance $r$ (col. [4]); slope $\alpha$ of the power-law continuum (col. [5]); observed value of $\theta$ for the ionization cone (col. [6]); the predicted torus luminosity $L_{P}$ (col. [7]); and the observed IR luminosity $L_{\mathrm{IR}}$ corrected for contributions from the host galaxy (col. [8]).

\subsection{Error Estimates}

We also show in Table 2 some error estimates for the parameters obtained from the emission-line ratios. For $-\log (U)$, the second line for each entry shows the range of possible values considering the $[\mathrm{O} \mathrm{III}] \lambda 5007 / \mathrm{H} \beta$ and $[\mathrm{N} \mathrm{II}] \lambda 6583 / \mathrm{H} \alpha$ ratios. For $n$, we also show the range of gas densities given the errors in the $[\mathrm{S}$ II] $\lambda 6716 / \lambda 6731$ ratio. Finally, we show the resultant maximum range for $L_{P}$ due to these variations as the second entry in column (7).

\subsection{Validity of the Method}

To check the method used to calculate $L_{P}$, we have performed the calculation at several points in the ionization cones whenever possible. The predicted torus luminosity should not depend on where the emission-line ratios are measured. In practice, several problems arise. First, the emission lines tend to get weaker as distance from the nucleus increases, leading to larger uncertainties in the estimates of $U, n$, and thus $L_{P}$. The second complication is that the very extended gas is often in

TABLE 2

Measured and Calculated Properties

\begin{tabular}{|c|c|c|c|c|c|c|c|}
\hline $\begin{array}{l}\text { Galaxy } \\
\text { (1) }\end{array}$ & $\begin{array}{l}r(\mathrm{pc}) \\
(2)\end{array}$ & $-\underset{\text { (3) }}{-\log (U)}$ & $\begin{array}{c}n\left(\mathrm{~cm}^{-3}\right) \\
(4)\end{array}$ & $\begin{array}{c}\alpha \\
(5)\end{array}$ & $\begin{array}{c}\theta \\
(6)\end{array}$ & $\begin{array}{l}L_{P} \\
(7)\end{array}$ & $\begin{array}{l}L_{\mathrm{IR}} \\
(8)\end{array}$ \\
\hline NGC 1068 & 4144 & $\begin{array}{c}3.0 \\
2.9-3.1\end{array}$ & $\begin{array}{c}150 \\
50-250\end{array}$ & 1.6 & 40 & $\begin{array}{r}5.2 \times 10^{11} \\
(0.1-1.1) \times 10^{12} \\
(1.1-8.2) \times 10^{11}\end{array}$ & $9.6 \times 10^{10 a}$ \\
\hline NGC $1365 \ldots \ldots$ & 370 & $\begin{array}{c}3.1 \\
2.9-3.4\end{array}$ & $\begin{array}{c}150 \\
50-250\end{array}$ & 1.5 & 90 & $\begin{array}{r}2.1 \times 10^{9} \\
(0.4-5.6) \times 10^{9}\end{array}$ & $9.8 \times 10^{9}$ \\
\hline NGC $2110 \ldots \ldots$ & 508 & $\begin{array}{c}2.7 \\
2.5-2.9\end{array}$ & $\begin{array}{c}130 \\
50-200\end{array}$ & 1.5 & 30 & $\begin{array}{r}1.1 \times 10^{10} \\
(0.3-2.8) \times 10^{10} \\
(0.8-3.5) \times 10^{10}\end{array}$ & $8.4 \times 10^{9}$ \\
\hline NGC $3281 \ldots \ldots$ & 770 & $\begin{array}{c}2.3 \\
2.2-2.4\end{array}$ & $\begin{array}{c}350 \\
300-400\end{array}$ & 1.5 & 70 & $\begin{array}{r}1.6 \times 10^{11} \\
(1.1-2.2) \times 10^{11} \\
(0.5-3.1) \times 10^{11}\end{array}$ & $2.0 \times 10^{10}$ \\
\hline NGC $4388 \ldots \ldots$ & 487 & $\begin{array}{c}2.6 \\
2.3-2.9\end{array}$ & $\begin{array}{c}250 \\
125-400\end{array}$ & 1.3 & $\begin{array}{l}92^{\mathrm{b}} \\
50^{\mathrm{c}}\end{array}$ & $\begin{array}{r}1.6 \times 10^{10} \\
(0.4-5.2) \times 10^{10} \\
(0.8-2.8) \times 10^{10}\end{array}$ & $4.6 \times 10^{9}$ \\
\hline NGC $5506 \ldots \ldots$ & 398 & $\begin{array}{c}2.8 \\
2.7-2.9\end{array}$ & $\begin{array}{c}400 \\
200-600\end{array}$ & 1.6 & 90 & $\begin{array}{r}1.5 \times 10^{10} \\
(0.6-2.8) \times 10^{10}\end{array}$ & $9.5 \times 10^{9}$ \\
\hline NGC $5728 \ldots \ldots$ & 879 & $\begin{array}{c}2.9 \\
2.8-3.0\end{array}$ & $\begin{array}{c}250 \\
100-400\end{array}$ & 1.5 & 50 & $\begin{array}{r}3.9 \times 10^{10} \\
(1.2-7.8) \times 10^{10}\end{array}$ & $1.4 \times 10^{10}$ \\
\hline Mrk $78^{d}$ & 5061 & $\begin{array}{c}2.5 \\
2.5-3.0\end{array}$ & $\leq 100$ & 2.0 & 50 & $\leq 3.2 \times 10^{12}$ & $5.7 \times 10^{10}$ \\
\hline Mrk $573 \ldots \ldots \ldots$ & 934 & $\begin{array}{c}2.9 \\
2.7-3.2\end{array}$ & $\begin{array}{c}800 \\
600-1000\end{array}$ & 0.9 & 80 & $\begin{array}{r}5.9 \times 10^{10} \\
(0.2-1.2) \times 10^{11} \\
(3-8.5) \times 10^{10}\end{array}$ & $1.6 \times 10^{10}$ \\
\hline
\end{tabular}

- Nuclear flux based on Telesco et al. 1984

bW cone (Pogge 1989).

c NE cone (Pogge 1989).

d In the low-density limit of the [S II] $\lambda \lambda 6716,6731$ density diagnostic. 
the low-density limit of the $[\mathrm{S}$ II] $\lambda 6716 / \lambda 6731$ line diagnostic and only an upper limit to the electron density can be found. For five galaxies we have calculated $L_{P}$ at several points where the density is above the low-density limit. In all cases, we find that $L_{P}$ is constant within the errors. The range of nominal values of $L_{P}$ found from considering several points in the cone is included in Table 2 (third entry under heading $L_{P}$ ). Finally, we note that the values of $L_{P}$ calculated for the two different ionization cones in NGC 4388 and Mrk 573 give very similar results.

\section{DISCUSSION}

The goal of our calculation is to test whether the IR emission from Seyfert 2's is consistent with collimation by a dusty torus. If the predicted torus luminosity is less than or equal to the observed IR luminosity then the observations are consistent with the model. If the predicted luminosity is significantly more than what is observed, either the ionizing source is collimated on much smaller scales or the IR flux is emitted anisotropically. This latter effect is possible because the torus may be optically thick even at mid-infrared wavelengths. Pier \& Krolik (1992) have modeled the reradiation from dusty tori and find that in edge-on systems (i.e. Seyfert 2's) the observed IR flux may be significantly less than the angle-averaged torus flux. The amount of anisotropy depends on the torus's optical depth and shape, but a factor of a few can be expected. Given such anisotropy and the considerable uncertainties in the predicted torus luminosity and the nuclear contribution to the IRAS fluxes, values of $L_{P}$ that are a few times larger than $L_{\mathrm{IR}}$ must be considered consistent with the torus model.

A comparison of $L_{P}$ and $L_{\mathrm{IR}}$ from Table 2 shows that for six of the sample galaxies the agreement between these two quantities is very good. A plot of $L_{P}$ versus $L_{\mathrm{IR}}$ (Fig. 1) is largely consistent with a linear relation between these quantities. For a few galaxies, the nominal value of $L_{P}$ is larger than $L_{\mathrm{IR}}$, but when the errors are taken into account, a significant discrepancy (a factor of 8) exists for only NGC 3281. Such an excess of $L_{P}$ over $L_{\mathrm{IR}}$ may be consistent with the torus model if the torus emission is significantly anisotropic in the mid-infrared. In NGC 1068, for example, there are some indications that such may be the case. First, the hard X-ray observations imply a very large column density through the torus $\left(N_{\mathrm{H}} \geq 10^{25} \mathrm{~cm}^{-2}\right.$ : cf. Mulchaey, Mushotzky, \& Weaver 1992a). For columns this large, the IR emission is significantly anisotropic even at $60 \mu \mathrm{m}$ (Pier \& Krolik 1992). Second, the large extent of the highexcitation emission-line gas in NGC 1068 suggests we may be seeing the torus edge-on, which decreases the observed IR flux from its value averaged over all possible directions of view (Pier \& Krolik 1992). Thus, a strong case can be made that the ratio of $L_{P}$ to $L_{\mathrm{IR}}$ in NGC 1068 is consistent with the torus model. For NGC 3281, there are no hard X-ray data, but here

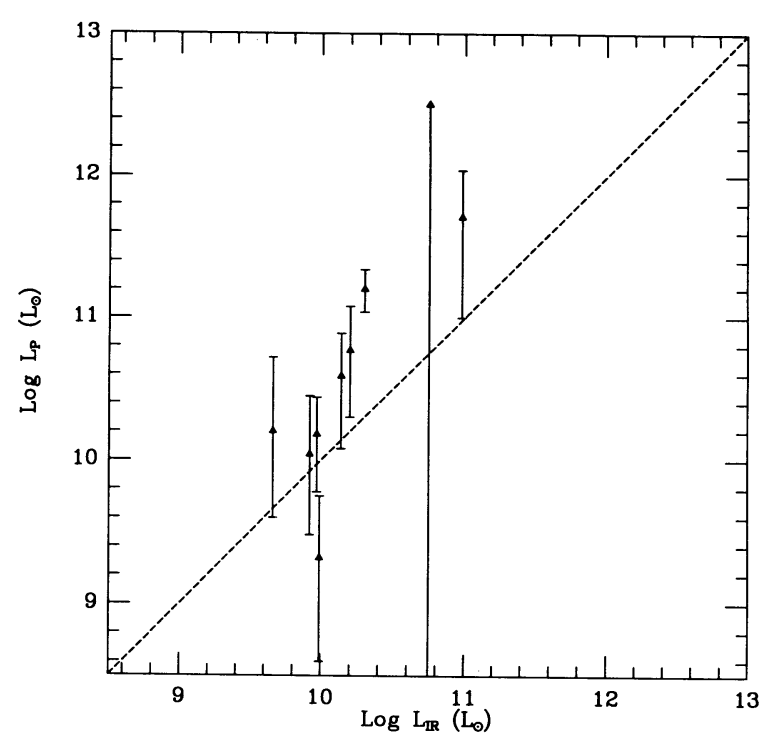

Fig. 1.-A logarithmic plot of $L_{P}$ vs. $L_{\mathrm{IR}}$

too the torus may be seen nearly edge-on (Storchi-Bergmann, Wilson, \& Baldwin 1992), and significant anisotropy in the mid-infrared might be present. Alternatively, the central ionizing source itself could radiate anisotropically.

\section{CONCLUSIONS}

We have used long-slit and imaging data on a sample of nine nearby Seyfert 2 galaxies with ionization cones in order to test a prediction of the "Unified Scheme," namely that these galaxies contain a compact nucleus obscured by a dusty torus. From the observed emission-line ratios and opening angles of the ionization cones, the luminosity incident on the torus was calculated. On the assumption that the compact ionizing source is isotropic and that the torus absorbs all the radiation with wavelengths between $100 \AA$ and $1 \mu \mathrm{m}$ and reemits in the infrared, we have compared this predicted luminosity $L_{P}$ with the observed IR luminosity $L_{\mathrm{IR}}$ calculated from the IRAS fluxes.

For all but one object, the predicted and observed luminosities agree to within the errors. We conclude, therefore, that the data are generally consistent with collimation and reradiation by a dusty torus.

We acknowledge Gary Ferland, Julian Krolik, Ed Pier, and the anonymous referee for valuable suggestions. T. S. B. acknowledges a fellowship from the Brazilian Institution CNPq. This research was supported by NASA grants NAG51079, NAG5-1886, NAGW-2689, and NAG8-793 to the University of Maryland.

\section{REFERENCES}

Acosta-Pulido, J. A., Pérez-Fournon, I., Calvani, M., \& Wilson, A. S. 1990 , ApJ, 365, 119

Antonucci, R. R. J., \& Miller, J. S. 1985, ApJ, 297, 621

Baldwin, J. A., Wilson, A. S., \& Whittle, M. 1987, ApJ, 319, 84

Bergeron, J., Petitjean, P., \& Durret, F. 1989, A\&A, 213, 6

Colina, L. 1992, ApJ, 386, 59

Corbin, M., Baldwin, J. A., \& Wilson, A. S. 1988, ApJ, 334, 584

Edmunds, M. G., \& Pagel, B. E. J. 1982, MNRAS, 198, 1089

Ferland, G. J. 1991, OSU Internal Report, No. 91-01

Haniff, C. A., Wilson, A. S., \& Ward, M. J. 1991, ApJ, 368, 168

Kinney, A. L., Antonucci, R. R. J., Ward, M. J., Wilson, A. S., \& Whittle, M. 1991, ApJ, 377, 100

Krolik, J. H., \& Begelman, M. C. 1986, ApJ, 308, L55

Krolik, J. H., \& Lepp, S. 1989, ApJ, 347, 179

Madau, P. 1988, ApJ, 327, 116

Miller, J. S., Goodrich, R. W., \& Mathews, W. G. 1991, ApJ, 378, 47

Miley, G. K., Neugebauer, G., \& Soifer, B. T. 1985, ApJ, 293, L11

Mulchaey, J. S., Mushotzky, R. F., \& Weaver, K. A. 1992a, ApJ, 390, L69

Mulchaey, J. S., Wilson, A. S., \& Whittle, M. 1992b, in preparation

Mulchaey, J. S., et al. 1992c, in preparation

Pedlar, A., Meaburn, J., Axon, D. J., Unger, S. W., Whittle, D. M., Meurs, E. J. A., Guerrine, N., \& Ward, M. J. 1989, MNRAS, 238, 263

Pier, E., \& Krolik, J. H. 1992, ApJ, submitted.

Phillips, M. M., Turtle, A. J., Edmunds, M. G., \& Pagel, B. E. J. 1983, MNRAS, 203, 759

Pogge, R. W. 1988a, ApJ, 328, 519 
Pogge, R. W. 1988b, ApJ, 332, 702

. 1989, ApJ, 345, 730

Roche, P. F., Aitken, D. K., Smith, C. H., \& Ward, M. J. 1991, MNRAS, 240, 838

Rowan-Robinson, M., \& Crawford, J. 1989, MNRAS, 238, 523

Schommer, R. A., Caldwell, N., Wilson, A. S., Baldwin, J. A., Phillips, M. M., Williams, T. B., \& Turtle, A. J. 1988, ApJ, 324, 154

Storchi-Bergmann, T., \& Bonatto, C. J. 1991, MNRAS, 250, 138

Storchi-Bergmann, T., Wilson, A. S., \& Baldwin, J. 1992, in press
Telesco, C. M., Becklin, E. E., Wynn-Williams, C. G., \& Harper, D. A. 1984, ApJ, 282, 427

Tsvetanov, Z., \& Walsh, J. W. 1992, ApJ, 386, 485

Veilleux, S. 1991, ApJ, 369, 331

Wilson, A. S. 1992, in Physics of Active Galactic Nuclei, ed. S. J. Wagner \& W. J. Duschl (Berlin: Springer-Verlag), in press

Wilson, A. S., Baldwin, J. A., \& Ulvestad, J. S. 1985, ApJ, 291, 627

Wilson, A. S., Ward, M. J., \& Haniff, C. A. 1988, ApJ, 334, 121 